\title{
Haptic processing of spatially distributed information
}

\author{
SUSAN J. LEDERMAN and ROGER A. BROWSE \\ Queen's University at Kingston, Kingston, Ontario, Canada \\ and \\ ROBERTA L. KLATZKY \\ University of California at Santa Barbara, Santa Barbara, California
}

\begin{abstract}
A haptic search paradigm, adapted from Treisman and Gelade's (1980) visual search tasks, was used as an initial step in addressing issues relevant to the development of models of human and machine haptic object processing. Texture and/or edge-orientation information were presented to multiple finger locations in disjunction (Experiment 1) and conjunction (Experiment 2) search tasks. In Experiment 3, subjects performed a difficult single-feature (orientation) search. Although the disjunction task could be interpreted with parallel or serial exhaustive models of haptic processing, subjects showed a shift toward serial self-terminating processing with the more complex and difficult tasks. These results indicate processing changes when features of texture and shape must be integrated. Given other converging evidence, texture may be a better candidate than edge orientation for early perceptual processing, with information being processed preattentively and in parallel.
\end{abstract}

Haptics is a perceptual system that provides both cutaneous and kinesthetic information through touching (Gibson, 1966; Loomis \& Lederman, 1986). Recent research by Klatzky, Lederman, and Metzger (1985) has shown that people are extremely accurate and very fast at haptically identifying three-dimensional common objects. Such work serves as an existence proof that the human haptic system can perform certain aspects of object processing surprisingly well. We believe it is therefore important to study the nature of the haptic representation of objects in memory and the underlying processes by which those representations are achieved and utilized. Such an undertaking serves a more applied purpose as well. It has recently been documented (Harmon, 1982) that robotic perceptual systems involving feedback from tactile sensors (e.g., the use of pressure information obtained from an array of artificial skin sensors) may play an important role in the automated industry of the future. Results from biological haptic research may further serve as a valuable guide in the development of models of machine object processing.

Although haptic object exploration tends to be extended in time, more than one finger may be used simultaneously during the exploratory process. Furthermore, Lederman

This research was supported by grants to S.J.L. and R.A.B. from the Natural Sciences and Engineering Research Council of Canada. We would like to thank Andy Currie for his considerable assistance and input in running the experiments, and Lester Krueger, as consulting editor, for his insightful comments. Reprints may be requested from S. J.Lederman (Department of Psychology), R. A. Browse (Department of Computing and Information Science), Queen's University, Kingston, Ontario, Canada K7L 3N6, or from R. L. Klatzky, Dept. of Psychology, University of California, Santa Barbara, CA 93106. and Klatzky have found, in work in progress, that the static grasp, involving the simultaneous use of several fingers of one or both hands, is a particularly effective and common means of recognizing objects. Accordingly, we are interested in the manner in which the haptic system processes simultaneous multiple inputs. To date, relatively little somatosensory research has addressed this issue. Studies by Shiffrin, Craig, and Cohen (1973), Lappin and Foulke (1973), and Craig (1985) have provided evidence that the haptic system is capable of at least limited parallel processing, that is, across several fingers within (but not across) hands. However, this work only begins to consider what is an enormously complex and important topic.

Vision research has much to offer the fledgling field of haptics, both with respect to perceptual theory and experimentation. For example, in a recent series of papers, Treisman (Treisman, 1986; Treisman \& Gelade, 1980; Treisman \& Paterson, 1984; Treisman \& Schmidt, 1982; Treisman, Sykes, \& Gelade, 1977) presented a specific analytic processing framework-feature integration theory-for visual attention, which distinguishes between preattentive and attentive processing. This theory states that individual features (e.g., a single color or form) may be processed preattentively, rapidly, and in parallel across a broad portion of the visual field, but that decisions based on conjunctions of features (e.g., color and form) normally require focused visual attention.

Do the types of processes that occur in early vision extend more generally to the haptic system? It is unlikely that the question will be answered completely in the affirmative, as there are some considerable differences between the two perceptual systems, the implications of which may be profound. First, for example, the systems 
are not equally sensitive to all forms of physical energy, nor are they likely to process the same attributes of objects and surfaces equally well. Second, the retina is an extended surface that moves as a unit, whereas the hand is a multi-degree-of-freedom structure with five appendages ("retinas"), which can be moved semi-independently during exploration. Third, while the haptic system commonly uses afferent information obtained through a sequence of contacts, vision usually has available for processing considerable information within a single retinal image.

It is clearly important to consider the ramifications of such differences for perceptual processing. On the other hand, these differences do not preclude the haptic system's resembling vision in having distinct preattentive and attentive encoding mechanisms, nor do they preclude its having identifiable features. Therefore, as a first exploratory step in studying these issues, it seemed worthwhile to begin by adapting a simple, but informative, visual paradigm as closely as possible for use with the haptic system.

Treisman has employed a number of experimental paradigms to test the validity of the feature integration theory. We chose to adopt the visual search paradigm, which is described next, for use with haptic displays. For vision, the search task tests the notion that, initially, an observer examines a visual display preattentively and without focused attention, analyzing the display in terms of various separable dimensions. Treisman et al. (1977) demonstrated that when searching for a colored target (pink) in a set of colored distractors (brown and purple) or a form target $(\mathrm{O})$ in form distractors $(\mathrm{N}$ or $\mathrm{T})$, the reaction time to determine that the target was present was independent of the number of items in the display or was nonlinear. Such flat or nonlinear target functions are interpreted in support of a parallel, preattentive search.

When subjects searched for the conjunction of values from two such separable dimensions (pink $O$ in green $O$ and pink $\mathrm{N}$ distractors), reaction time varied in a strictly linear fashion with the number of items in the display. Moreover, the ratio of the slope of the no-target to target functions was approximately $2: 1$, indicating the use of a serial self-terminating search. Treisman argued that, in the conjunction task, it is necessary to direct one's attento the location of a particular item in order to examine both of the properties at that position simultaneously.

Similar results (i.e., flat or nonlinear functions) were subsequently obtained with a more powerful disjunction task replacing the single-feature task (Treisman \& Gelade, 1980). Subjects were asked to search concurrently for either of two targets, each defined by a different feature (a form [S] and a color [blue]). The task therefore required the observer to attend to both dimensions at the same time (as in the conjunction condition); however, it was never necessary to consider the conjunction of these two dimensions.

Treisman's results are consistent with the notion that early visual processes are spatially parallel without ca- pacity limitations and that attention is required for spatial conjunction of distinct dimensions, although there are alternate interpretations of the reaction time functions (Townsend, 1974). Regardless of the underlying models, the search paradigm offers clear evidence of two types of visual processes, the operations of which depend upon the nature of the target dimensions.

We selected an equivalent haptic search paradigm since it allowed us to compare the processing of haptic inputs with existing visual data and interpretations. For example, we could examine the generality of the preattentiveattentive distinction by considering whether a similar processing distinction could be observed between disjunction and conjunction conditions in haptic search tasks. Texture and edge orientation were selected as stimulus dimensions on the basis of the physiological and behavioral data on the somatosensory system available at the time the project began (for reviews of this literature, see Loomis \& Lederman, 1986, and Sherrick \& Cholewiak, 1986). Textural tasks, in particular, are performed very well by the haptic system (e.g., Lamb, 1983; Morley, Goodwin, \& Darian-Smith, 1983); texture is also highly salient to haptics (Lederman, 1982; Lederman \& Abbott, 1981; and most recently, Klatzky, Lederman, \& Reed, 1987).

In keeping with Treisman and Gelade (1980), Experiment 1 involved a disjunction task in which observers searched each display for either a rough (horizontal) or a vertical (smooth) target, among smooth horizontal distractors. Experiment 2 involved a conjunction task in which observers looked for a rough and vertical target, among rough horizontal and smooth vertical distractors. Experiment 3 was conducted to consider whether the selfterminating process adopted in Experiment 2 (cf. Experiment 1) could be explained by an increase in task difficulty. It involved a difficult single-feature orientation task in which subjects searched each display for a slanted (smooth) target among (smooth) horizontal and (smooth) vertical distractors. The stimulus displays in all experiments varied in the number of items present.

\section{EXPERIMENT 1: HAPTIC SEARCH FOR TEXTURE/ORIENTATION DISJUNCTIONS}

In accord with the visual disjunction paradigm, observers searched for the presence of either a rough horizontal bar or a smooth vertical bar (target item), among smooth horizontal bars (distractor items). The target was present on only half of the trials. The number of items in the display varied from one to six. In any display, either no item (i.e., a smooth surface) or one item under each finger was possible. Observers judged whether the target was present ("yes") or not ("no") as quickly and as accurately as they could.

According to Treisman, if either texture and/or orientation is processed initially in parallel at a preattentive stage, the target function (reaction time as a function of number of items) should be flat; that is, reaction time 
should be relatively unaffected by the number of items in the stimulus displays. Alternatively, the functions may contain a significant nonlinear component, although Treisman argues that the latter does not imply a serial decisionmaking process. The interpretation of visual nonlinearities is discussed briefly in the Discussion section following Experiment 2 (see also Treisman \& Gelade, 1980, p. 112).

Serial processing would be indicated by nonflat target and no-target functions in which only the linear component was statistically significant. In this event, the ratio of the no-target to target slopes would further suggest the nature of the serial process. For example, parallel functions would indicate an exhaustive search, whereas a 2:1 ratio would point to a serial self-terminating search. Variability can be used in addition to make this distinction (Townsend \& Ashby, 1983). Because of the variation in termination points, self-terminating search should have greater variance than exhaustive search.

\section{Method}

Observers. A total of 8 observers, 3 females and 5 males, were paid for their participation. Their ages ranged from 19 to 37 , with a mean of 24 years. All were experimentally naive and described themselves as right-handed.

Apparatus and Stimuli. The stimulus items were threedimensional bars ( $17 \mathrm{~mm}$ long $\times 3 \mathrm{~mm}$ wide $\times 2 \mathrm{~mm}$ high) constructed of Plexiglas with a covering surface of plastic Brailon paper. Two dimensions of each bar were independently varied: texture and orientation. Thus, a bar could have a smooth or rough surface texture, and it could also be oriented either vertically or horizontally.

The smooth surface simply consisted of unengraved plastic paper, cut to size, and glued to the top surface of the three-dimensional bar. The rough surface was created by engraving a regular raiseddot pattern on plastic paper. The engraving was achieved by a process known as thermoforming, in which heat-sensitive plastic paper is melted over a three-dimensional pattern master and drawn down by a vacuum pump to conform to the pattern beneath. The masters were created for other experiments by producing regular print (i.e., two-dimensional) arrays of dots by computer, and then photoengraving the patterns, using a Nyloprint technique to produce plastic polymer masters. When cut to cover the top of the rectangularly shaped bar, the dots (approximately $0.4 \mathrm{~mm}$ in diameter) formed a single line along the long dimension of the bar with a 2-mm separation between the inner edges of adjacent dots. However, when examined haptically, the bar appeared to have a uniformly rough surface texture with no property of orientation independent of that of the bar (i.e., the set of engraved dots was not perceived as a line superimposed within and parallel to the long edges of the bar).

The orientation dimension consisted of two feature values: vertical and horizontal; that is, the bars were oriented either parallel or perpendicular to the observer's finger axis. Pilot work confirmed that the texture and the orientation pairs were both clearly discriminable.

The apparatus is shown in Figure 1. It consisted of two Plexiglas three-finger slots (one for each hand), a display tray, and the stimulus display sheets. The finger slots were $7.7 \mathrm{~cm}$ across, $1.2 \mathrm{~cm}$ high, and $6.4 \mathrm{~cm}$ deep. Each separate finger trough was $1.8 \mathrm{~cm}$ across, $0.9 \mathrm{~cm}$ high, and $2.5 \mathrm{~cm}$ deep. The closed ends were semicircular to fit the natural curve of the fingertips; the middle finger troughs were offset an additional $0.5 \mathrm{~cm}$ to adjust for their relatively longer length.
The display tray was constructed of Masonite. The base of the tray measured $28.0 \mathrm{~cm}$ across $\times 12.0 \mathrm{~cm}$ deep $\times 0.1 \mathrm{~cm}$ thick, with $26.0 \times 1.0 \times 0.1 \mathrm{~cm}$ edges added to the top of the front and back sides of the tray. The left and right edges were $1 \mathrm{~cm}$ across $\times 12 \mathrm{~cm}$ deep $\times 0.2 \mathrm{~cm}$ thick. These edges permitted the experimenter to insert the $26 \times 10 \mathrm{~cm}$ display sheets into the correct position with respect to the observer's fingers. The stimulus sheets were fixed in place by two-sided tape placed on the stimulus tray. There were holes in the base of the tray to assist the experimenter in removing the stimulus sheets quickly. The tray was attached to and balanced between the pads of two Armaco Brass telegraph keys, one centered under each hand.

There were six stimulus positions on the stimulus displays, each measuring $2 \times 2 \mathrm{~cm}$. Numbering them from 1 to 6 , corresponding to left ring finger, left middle, left index, right index, right middle, and right ring, positions $1,3,4$, and 6 were $0.25 \mathrm{~cm}$ above the horizontal midline and $1.75 \mathrm{~cm}$ below it. Positions 2 and 5 were $1.75 \mathrm{~cm}$ above the midline and $0.25 \mathrm{~cm}$ below it. The locations of the stimulus positions, given as the distance in centimeters from the left-hand edge of the sheet to where each began, were 3.3, 5.8, $8.8,16.3,18.8$, and $21.3 \mathrm{~cm}$ for Positions 1 to 6 , respectively.

In any stimulus display, the target was either a rough (horizontal) or a vertical (smooth) bar, but never both. The distractors were all smooth horizontal bars.

Two sets of 12 different displays were prepared for use in the texture trials; another two sets of 12 displays were prepared for the orientation trials. Each display consisted of from one to six items and, over a set of 12 displays, each possible number of items was presented both with and without a target (target and no-target conditions, respectively); the target was presented once in each of the six finger positions. The position of the distractors was determined randomly, with the stipulation that they be relatively equally distributed across each finger position. Note then that, on each trial, it was necessary to monitor the three fingers of both hands. A set of practice displays was also prepared for training the observers on the texture-orientation disjunction task.

A VK-003 voice key, a Vantek V-2600 Unidirection Dynamic microphone, and a DEC PDP-11/03 computer were used to collect the reaction times by monitoring the voice and telegraph-key responses via a parallel interface.

Procedure and Experimental design. Each observer read the following instructions: "On each trial, you will feel raised stimuli under some of your fingers. You are asked to determine if any one of those stimuli is one of the specified targets (ROUGH or VERTICAL). The remaining stimuli will be HORIZONTAL and SMOOTH. For any given trial there will be only one target (either ROUGH or VERTICAL, but not both), if it appears at all. The target will only be present on half the trials. You are asked to state if the target is present ('yes' or 'no'). Do this as quickly as possible since speed is being measured. However, it is also EXTREMELY IMPORTANT that you make no mistakes in this task. You will be told whether you are correct or not after each trial. Please do not use your nails."

The observer was shown the apparatus and then blindfolded for the remainder of the session. Next, the observer practiced sliding both hands away from the finger slots so that all the fingers would contact the appropriate positions in the stimulus display at approximately the same time. Once contact was made, the observer could move fingers in any direction over the stimuli.

The observer was instructed to determine whether there was a target present or not on each trial. Although the observer was aware that the total number of items in a display could vary from one to six, he or she was never told the precise number on any trial. The observer was told to answer "yes" when there was a target and "no" when there wasn't one, and was instructed to be as fast and as accurate as possible. On each trial, the experimenter indicated whether the answer was correct or not. If an error was made, the 

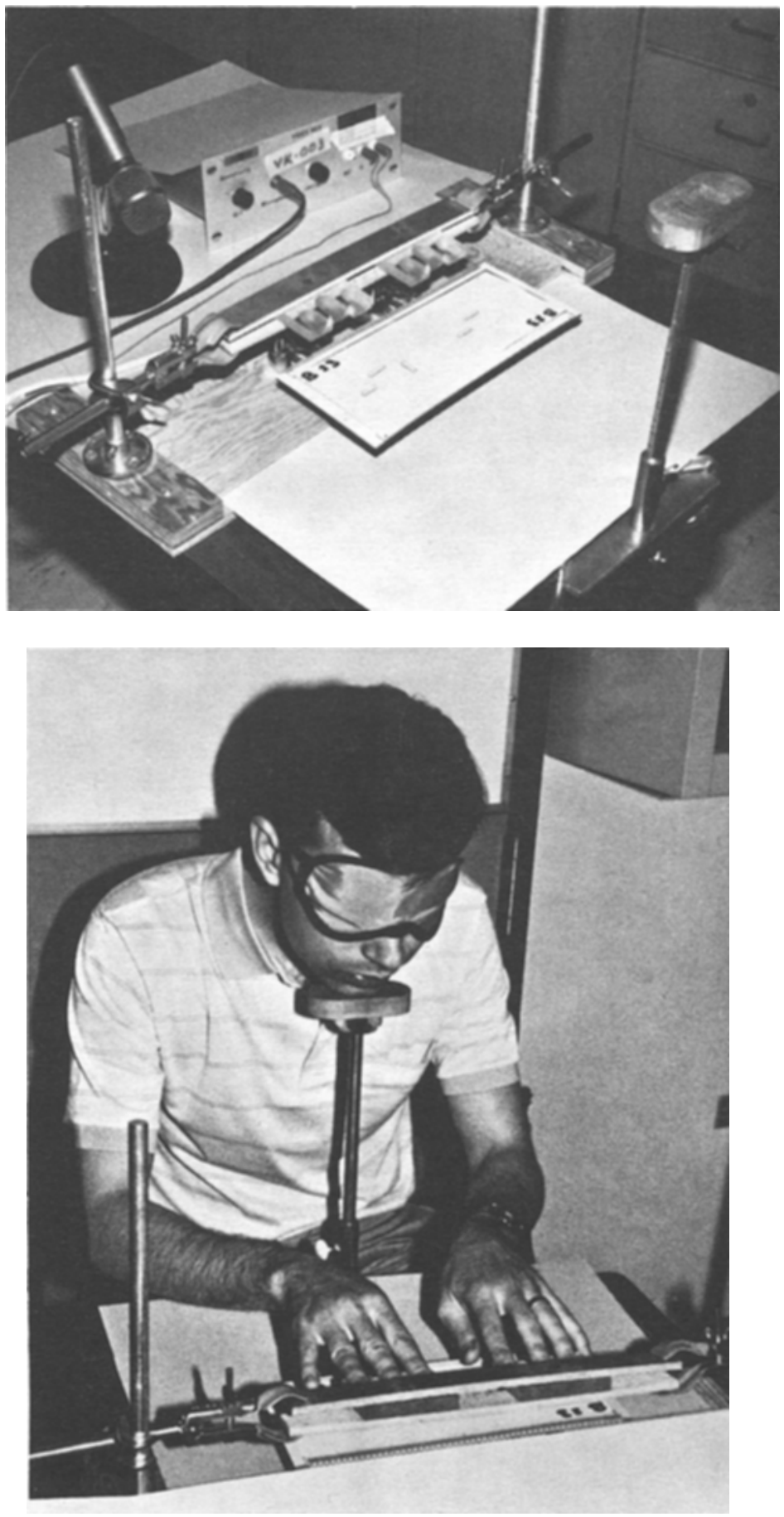

Figure 1. The experimental apparatus.

trial was repeated at the end of the session without informing the observer. Thus, only the reaction times for correct trials were used in the statistical analyses.

The observers began with at least eight practice trials. On each trial, the stimulus display was placed in the tray while the observer waited with hands pulled back from it. When told by the experimenter to do so, the observer placed both hands in the finger slots. On a second signal, the observer moved the hands down to the stimulus tray, with the middle three fingers of both hands guided to Stimulus Positions 1 through 6. As soon as the tray was depressed, that is, when the switches to both telegraph keys had been triggered, the computer timer began. The observer was allowed to examine each item only with the associated finger. The trial terminated when the observer said "yes" or "no"; activation of the voice key stopped the timer. The reaction times were recorded by the computer.

Next, the observers were given four complete blocks of trials, each presenting the full set of $\mathbf{4 8}$ experimental displays in a different random order. A 5-min break, followed by eight practice trials, 
occurred between successive blocks. Thus, each observer received the same displays, but the order of presentation within each block was randomly varied. The first block served as additional practice, and was discarded. Across the three remaining blocks, the texture and orientation targets were presented in each of the one- to sixitem conditions a total of 6 times; the corresponding no-target conditions were each presented 12 times. As the stimulus displays and their presentation were not automated, it was virtually impossible to present the target in each of the six positions with all possible combinations of both number and position of the additional distractor items. Therefore, the current experimental design does not lend itself to an analysis of the relative performance of the different fingers, or to consideration of serial position effects.

\section{Results}

The reaction times were analyzed using an analysis of variance with a within-subject factorial design. The five factors were: target dimension (2), number of items (6), target/no-target (2), blocks (3), and replications within blocks (2).

The main effect of target dimension (orientation vs. texture) was not statistically significant. The main effect of number of items was highly significant $[F(5,35)=30.97$, $p<.0001]$. The effect of target/no-target was also statistically significant $[F(1,7)=13.45, p<.005]$. These effects may be seen in Figure 2, in which mean reaction times (for correct responses) are plotted as a function of the number of items in the display for the texture, orientation, and no-target conditions. The interaction term, number of items $\times$ target/no-target, failed to reach significance $[F(5,35)=0.96, p>.05]$. A linear trend analysis on the number of items indicated a significant linear component $[F(1,7)=70.41, p<.0002]$; however, the overall non-linear residual was also statistically significant $[F(4,28)=9.99, p<.001]$. For purposes of com- parison only, linear fits of the functions shown in Figure 2 were obtained with a least squares analysis. The slope: (millisecond increase in RT/added item) and corresponding $r^{2}$ values (bracketed) are as follows: no-target, 64 (.77); texture target, $65(.84)$; and orientation target, 55 (.50). The ratio of no-target to target slopes were $0.9^{\text {? }}$ and 1.08 for texture and orientation, respectively.

Of the remaining significant factors pertaining to the effects of repeating various stimulus conditions, only the main effect for blocks accounted for more than a negligible proportion of the variance $[F(2,14)=12.16$, $p<.005$ ], indicating that reaction times decreased with practice.

The standard deviations associated with the means for the 12 target/no-target $\times$ number of items conditions shown in Figure 2 are reported in Table 1. The error rates are also presented in Table 1: they averaged $1.5 \%$ and $5.5 \%$ for false positives and misses, respectively. The pattern of errors was for the false-positive rate to remain relatively constant; the miss rate, as a function of number of items, was somewhat variable. The finding that misse. exceed false alarms, especially as the display size increases, is quite typical of visual search data (e.g., Krueger \& Shapiro, 1980), and probably reflects the fact that nontargets outnumber targets (maximimum of one per display) in most displays.

To briefly summarize the results of Experiment 1, the no-target and two-target functions for the disjunction task were parallel, as indicated by the nonsignificant double (number of items $\times$ target/no-target) and triple interaction terms (items $\times$ target/no-target $x$ target dimension), and by the fact that with linear fits to these functions, the ratios of the slopes for no-target to target functions were close to $1: 1$, for both texture and orientation. The slopes

Table 1

\begin{tabular}{|c|c|c|c|c|c|c|c|}
\hline \multirow{2}{*}{$\begin{array}{l}\text { Number } \\
\text { of Items }\end{array}$} & \multicolumn{3}{|c|}{$\begin{array}{l}\text { Experiment } 1 \\
\text { (Disjunction) }\end{array}$} & \multicolumn{2}{|c|}{$\begin{array}{l}\text { Experiment } 2 \\
\text { (Conjunction) }\end{array}$} & \multicolumn{2}{|c|}{$\begin{array}{c}\text { Experiment } 3 \\
\text { (Single Feature-Orientation) }\end{array}$} \\
\hline & No Target & Target $(\mathrm{T})$ & Target $(\mathrm{O})$ & No Target & Target & No Target & Target \\
\hline \multicolumn{8}{|c|}{ Standard Deviations (seconds) } \\
\hline 1 & 120 & 91 & 149 & 137 & 110 & 437 & 462 \\
\hline 2 & 161 & 101 & 143 & 271 & 133 & 663 & 777 \\
\hline 3 & 169 & 145 & 231 & 422 & 293 & 985 & 523 \\
\hline 4 & 236 & 212 & 324 & 286 & 446 & 514 & 759 \\
\hline 5 & 227 & 212 & 200 & 367 & 189 & 896 & 1044 \\
\hline 6 & 183 & 202 & 220 & 292 & 322 & 1188 & 981 \\
\hline Mean $S D$ & 183 & 161 & 211 & 296 & 249 & 781 & 758 \\
\hline \multicolumn{8}{|c|}{ Errors (\%) } \\
\hline 1 & 3.0 & 0.0 & 0.0 & 2.0 & 0.0 & 0.0 & 13.5 \\
\hline 2 & 5.0 & 4.0 & 15.8 & 2.0 & 4.0 & 3.0 & 13.5 \\
\hline 3 & 4.0 & 2.0 & 2.0 & 0.0 & 18.6 & 5.9 & 13.5 \\
\hline 4 & 5.0 & 17.2 & 22.6 & 2.0 & 9.4 & 8.6 & 31.9 \\
\hline 5 & 5.0 & 29.4 & 4.0 & 7.7 & 15.8 & 8.6 & 15.8 \\
\hline 6 & 3.0 & 15.8 & 12.7 & 4.0 & 12.7 & 20.0 & 30.4 \\
\hline \multirow[t]{2}{*}{ Mean errors } & 4.2 & 11.4 & 9.5 & 3.0 & 10.1 & 7.7 & 19.8 \\
\hline & FA & miss & miss & FA & miss & FA & miss \\
\hline
\end{tabular}

Note $-F A=$ false alarms, $T=$ texture, $O=$ orientation. 


\section{DISJUNCTION}

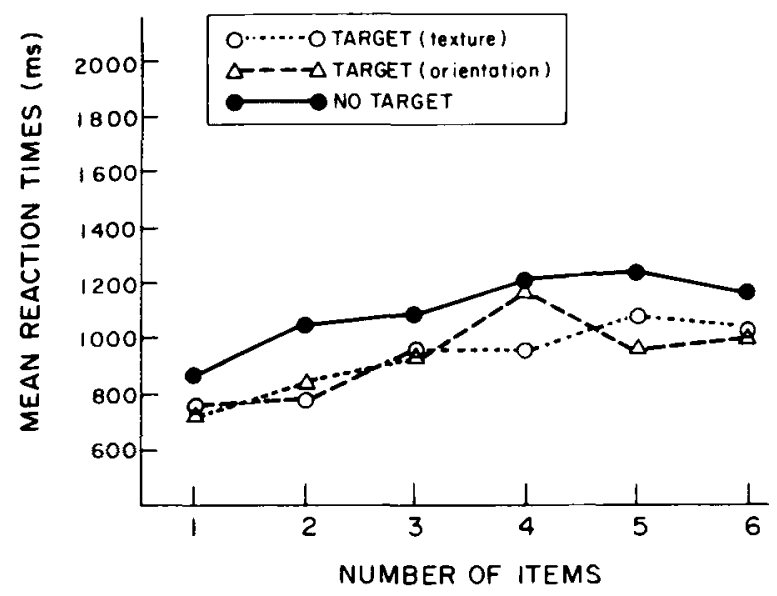

Figure 2. Experiment 1: Mean search times as a function of number of items in the stimulus display (disjunction task).

were substantially greater than zero, indicating increasing (not flat) functions. Finally, there were significant nonlinearities in the functions. The results will be discussed at the end of Experiment 2.

\section{EXPERIMENT 2: HAPTIC SEARCH FOR TEXTURE/ORIENTATION CONJUNCTIONS}

In Experiment 2, observers were instructed to search for a conjunction target, namely a rough and vertical bar, among smooth vertical and rough horizontal distractors. Treisman has argued that to the extent that the conjunction target and no-target functions are completely linear and not flat, the visual conjunction task is performed using a serial search strategy. The nonlinearities observed in the disjunction search should not occur, since only one item at a time is being searched. To the extent that a notarget:target-slope ratio of 2:1 occurs, the data would further support the interpretation that a self-terminating strategy has been adopted, in which observers stop as soon as they find a target.

\section{Method}

Observers. Eight undergraduate observers, 5 males and $3 \mathrm{fe}$ males, ranging in age from 18 to 25 years, with a mean of 20.6 years, participated. They were paid $\$ 5$. All described themselves as right-handed and were experimentally naive.

Apparatus and Stimuli. The apparatus and method of preparing stimulus items and displays are discussed in detail in Experiment 1 . Two dimensions were used to form conjunctions, namely, texture and orientation.

The texure dimension consisted of two features: roughness and smoothness. The orientation dimension consisted of two features: vertical and horizontal, that is, either parallel or perpendicular to the observer's finger axis.

The target items were rough and vertical. The two kinds of distractors were rough and horizontal, and smooth and vertical. There were 12 practice and 24 (i.e., two different sets of 12) experimental stimulus displays used. For each set of 12 displays, the target was present on half. Each display consisted of from one to six items, each possible number of items being presented both with and without a target. The target was presented once in each of the six finger positions per set. The position of the distractors was determined randomly, with the stipulation that the two kinds of distractors be relatively equally distributed across each finger position over the entire set of stimulus displays. As mentioned in Experiment 1, the experimental design chosen to accommodate the limitations of preparing and presenting the stimulus displays manually does not lend itself to analysis of either relative finger performance or serial position effects.

Procedure and Experimental design. Each observer read a set of instructions and was shown the apparatus. He or she was then blindfolded for the remainder of the session. As in Experiment 1, the observer practiced sliding both hands away from the finger slots to the stimulus display below. The instructions were identical to those used in Experiment 1, except that the observers were told to search for the presence of a rough and vertical target from among rough and horizontal, and smooth and vertical, distractor items.

The observers began with the practice set presented twice, for a total of 24 trials. Following the practice trials, the 24 experimental stimulus displays were presented three times to each observer. The order of presentation within each block of 24 trials was randomly determined within and across observers. The average experimental session lasted about $45 \mathrm{~min}$. There was a break of 3-5 min between experimental blocks.

\section{Results}

The reaction times for correct responses were analyzed by analysis of variance using a factorial design with four within-subject factors: number of items (6 levels), target/no-target (2), blocks (3), and replications within blocks (2).

The main effect for number of items was highly significant $[F(5,35)=32.8, p<.0001]$. The main effect for target/no-target was significant $[F(1,7)=19.9$, $p<.005]$, indicating that the no-target items took longer to evaluate than the target items. These effects may be seen in Figure 3: the mean (correct) reaction times are plotted as a function of the number of items for both the target and no-target conditions. Each datum is based on

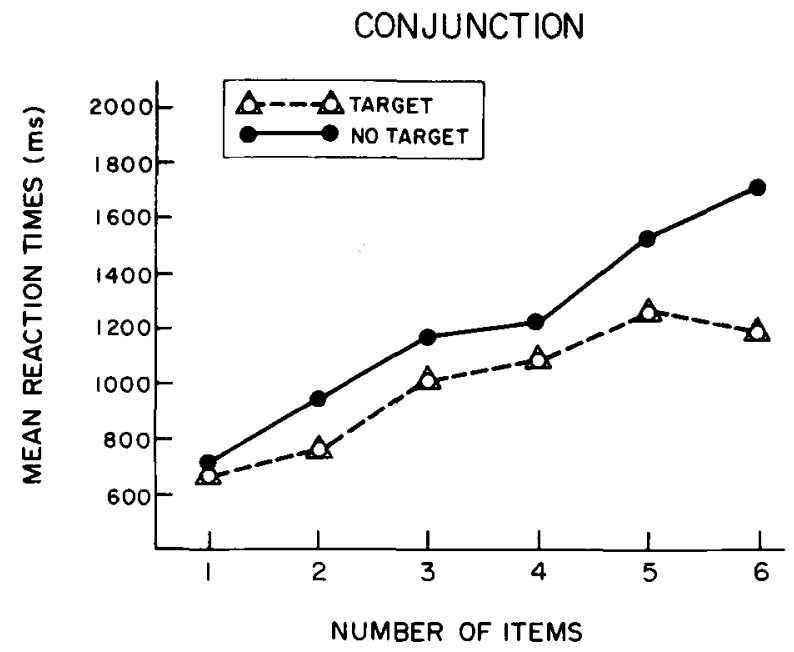

Figure 3. Experiment 2: Mean search times as a function of number of items in the stimulus display (conjunction task). 
48 scores, with one exception. Due to an error, the data point representing the no-target, 3 -item condition is based on 45 scores. The interaction of these two terms was also highly significant $[F(5,35)=5.1, p<.005]$. Thus, we examined the results of separate trend analyses on the number-of-items term for target and no-target functions. For the target function, the linear component was highly significant $[F(1,7)=28.17, p<.002]$; the residual nonlinear component barely attained significance $[F(4,28)=$ $2.72, p=.05]$. For the no-target function, only the linear component was significant $[F(1,7)=86.06, p<.0003]$; the nonlinear residual component was not significant $[F(4,28)=1.65, p>.10]$.

A least squares analysis yielded slopes (msec/item) of $194\left(r^{2}=.98\right)$ and $119\left(r^{2}=.88\right)$ for the no-target and target functions, respectively, with a ratio of 1.63. A $t$ test was performed on the corresponding slope ratios for the 8 individual subjects. The ratio of the no-target to target slopes was not statistically different from $2.0[t(7)=$ 1.48, $p>.05]$.

The standard deviations and errors associated with the 12 target/no-target $\times$ number of items conditions are reported in Table 1. Error rates were approximately $4 \%$ and $10 \%$ for the false positives and misses, respectively. The error rates for the false positives were again relatively low; there was no obvious pattern for the target data.

\section{Discussion of Experiments 1 and 2}

The following discussion is exploratory and speculative in nature. At this early stage in our research program, we view our results more as a vehicle for probing critical issues likely to prove important in subsequent investigation than as confirmation of specific perceptual processes. We have already noted several differences between the visual and haptic systems that may affect perceptual processing. In addition, when applying tests of preattentive/attentive and parallel/serial processing to the haptic domain, there are also considerable constraints on the amount of data that can be collected manually. This is partly due to the lack of a suitable technology for quickly and simultaneously presenting to the skin patterns that can be dynamically controlled in three dimensions. It is also partly due to the inherently slow, sequential nature of much haptic exploration, that is, of finger and hand movements. Accordingly, our data base is one-quarter the size of Treisman and Gelade's (1980, Experiment 1), and hence considerably more variable.

Recognizing these experimental limitations, let us consider the results of the haptic conjunction task first. The major results are that the no-target and target functions are not parallel, as indicated by the significant interaction term, items $\times$ target/no-target. The slope ratio for the no-target:target functions is not statistically different from 2.0. Finally, the residual nonlinear component of the no-target function is not significant; for the target function, it barely attains significance at the .05 alpha level. The mean reaction time data are very similar to those ob- tained by Treisman and her colleagues in visual conjunction searches, and would seem to indicate that a serial, self-terminating search is being performed under corresponding haptic search conditions as well, using focused attention.

Krueger (1984) has considered two additional indicators of self-termination in a same/different visual search task in which display length was varied. The indicators are the standard deviations of the reaction times and the error rate. He noted two competing influences of selfterminating processing on the standard deviation (Townsend \& Ashby, 1983, Proposition 7.7). First, the variability of reaction times should generally be increased under self-termination, because there is a greater range of places to stop processing. This is a "macro" level effect of the search process as a whole. Second, and contrary to the first effect, variability should be decreased, because each item's individual variability contributes to the whole, and fewer items are processed on average in a selfterminating process. This is a "micro" effect at the item level. The presence of two competing effects means that self-terminating processing will lead to a larger standard deviation than exhaustive processing only if the macrolevel effect is large relative to variability at the item level.

Micro effects might be expected to be quite high here, however, because of possible sources of variability that are unique to haptics. In particular, it is likely that subjects take some time to align their fingers on the individual items before processing of stimulus information can begin. Such an adjustment may explain the nonlinearities in the present disjunction task, assuming that alignment of several fingers can be done faster than each finger in isolation.

With these points in mind, consider the standard deviations in the current conjunction task. Here, early termination could occur on positive trials only if the target is found before the full set of items is processed. Negative, no-target trials clearly must constitute an exhaustive search. Note, too, that negative response times are generally larger, which would tend to increase variability. However, the standard deviation of the faster trials was as great as that of the no-target trials, particularly in the longest displays (where there are more possible termination points; see Townsend \& Ashby, Proposition 7.8). This suggests a mechanism that increases variability in the target condition, namely, self-termination.

Krueger (1984) also pointed out that error data might reflect the processing strategy underlying reaction times. The data for the conjunction task show a pattern consistent with Krueger's prediction for self-termination, in that the miss (false-negative) rate tends to increase with display size. More definitive evidence would be an increase in errors when the target position moves from left to right in the display, but, because of the experimental constraints mentioned at the beginning of this section, it was not possible to obtain reliable serial position data.

In the disjunction task, the target and no-target functions are not flat. The ratio of the no-target to target slopes is close to 1:1 (as indicated by the fact that the appropri- 
ate interaction is not statistically significant). These results differ from those of Treisman and her colleagues, who have obtained relatively flat target functions and very large no-target to target slope ratios (e.g., 8:1), which they interpret as evidence of an unlimited-capacity parallel search.

There are at least two interpretations of the disjunction data in Experiment 1 that we have seriously considered. The first alternative, which is consistent with Treisman's visual disjunction model, suggests that the haptic disjunction task in Experiment 1 is performed by an unlimitedcapacity parallel process that occurs preattentively. It suggests that the positive slopes of the two parallel functions, and the nonlinearities (as noted above), are produced by a serial multiple-finger alignment process superimposed on an unlimited-capacity parallel decision process. We propose that the serial process is the result of subjects' having to ensure that all fingers are properly aligned on each item. Note that the early multiple-finger coordination process proposed is unrelated to the processes determined by the decisions about item dimensions. This interpretation, then, is consistent with Treisman's argument that the visual nonlinearities she obtained in the disjunction search task were due to serial eye fixations.'

In summary, this first interpretation suggests that in the disjunction task, the target and no-target functions represent the results of a capacity-unlimited parallel search, while the properties of the conjunction functions indicate a serial self-terminating process. In keeping with this Treisman interpretation, the fact that the reaction times for one-item searches are identical in both the disjunction and conjunction search tasks is, as Treisman argues (Treisman \& Gelade, 1980, p. 105), critical to the preattentive/attentive distinction in visual data. It is only when the observer must process information presented to multiple spatial positions that we would expect differences in reaction time as a function of the nature of the search task.

A second alternative is that a serial, but exhaustive, search underlies the parallel disjunctive functions obtained, in contrast to the serial self-terminating search proposed for the conjunction task. Sternberg (1975) has argued that processing in memory search shifts from serial exhaustive to self-terminating when the difficulty of the task increases. Although the argument is initially counterintuitive, an exhaustive search may, in fact, prove more expedient than a serial, self-terminating search if the time taken to make a comparison between each item and the memory of the target is fast, relative to the time required to decide if there is a match. If we were to extend this interpretation to the haptic tasks, the difference between Experiments 1 and 2 could then be explained as a change in serial processing from exhaustive to self-terminating, due to an increase in task difficulty (and concomitant slowed search rate), rather than as a change in processing that switches from not requiring to requiring attentive capacity. Experiment 3 was therefore performed to assess the consequence of manipulating task difficulty directly.

In a final comment on Experiments 1 and 2, we note that the tendency of the no-target function to lie above the target function agrees with other studies which have found that it takes longer to say "no" than to say "yes" (e.g., Nickerson, 1966). The difference in reaction time between the two functions may simply be due to the fact that less acoustic energy is required to say "no" than "yes" (although it is sometimes found with digit-press as well). The results of an additional small pilot study support this interpretation: Two observers were required to drop their fingers from the finger holder onto the display below and to say "yes" or "now" (rather than "no") as soon as their fingers made contact. A number of displays with 1-6 items were presented. As with the "no" curves, the "now" curves lay consistently above the "yes" curves.

\section{EXPERIMENT 3: PROCESSING A DIFFICULT HAPTIC SINGLE-FEATURE (ORIENTATION) SEARCH}

Manipulating task difficulty directly provides a means of evaluating the issues raised above. In keeping with Sternberg's (1975) argument, a difficult haptic task with a slow rate of item processing should force a serial selfterminating process, as with memory search tasks using visual probes. If so, we should find a no-target:targetslope ratio similar to $2: 1$, as in Experiment 2 (conjunction task). This difficulty manipulation should also lead to reaction times, standard deviations, and errors that are higher than those of Experiment 1 (disjunction task).

These predictions were tested in Experiment 3, in which a single-feature (i.e., constant target) search was used. We had initially planned to increase the difficulty by adding intermediate values to both the orientation and texture dimensions. Unfortunately, it proved impossible to reproduce (by thermoforming) three precisely varying roughness values that yielded error rates low enough for reaction times to be interpretable. As there was no such difficulty with $0^{\circ}, 45^{\circ}$, and $90^{\circ}$ bar orientations, we chose to perform a single-feature (orientation) search experiment in which a bar slanted $45^{\circ}$ to the right served as target and (smooth) vertical and (smooth) horizontal bars served as distractors.

\section{Method}

Subjects. Eight undergraduates ( 4 males and 4 females) were paid for participating. Their mean age was 23.9 years; all described themselves as right-handed.

Stimuli and Apparatus. General details concerning stimuli and apparatus may be found in Experiment 1. The orientation dimension used in the single-feature search task consisted this time of three values: $0^{\circ}, 45^{\circ}$ (slanted right), and $90^{\circ}$.

The target was a (smooth) $45^{\circ}$ bar oriented to the right of the subject's longitudinal finger axis. The two kinds of distractors were (smooth) vertical and (smooth) horizontal bars. 
Procedure. Subjects were given the same kinds of instructions as previously. They began with a set of eight practice trials (four target and four no-target). This was followed by the presentation of a set of 24 stimulus displays, in random order, twice (i.e., two blocks). The set consisted of displays with one to six items, each possible number of items being presented twice within a block, both with and without a target. In each set of 24 displays, the target was presented twice in each of the six finger positions. However, as before, the position and type of distractor were determined randomly, such that the two distractors occurred about equally often within each display as well as across the set of 24 displays.

\section{Results and Discussion}

The reaction times were analyzed in an analysis of variance using a factorial design with four within-subject factors: number of items (6), target/no-target (2), blocks (2), and replications within blocks (2).

Figure 4 shows the mean reaction times for the 12 number of items $\times$ target/no-target conditions. The main effect for number of items was highly significant $[F(5,35)$ $=9.00, p=.0001]$. As in Experiment 2, a trend analysis was performed separately on the target and no-target functions, using the number-of-items term. Only the linear components were significant $[F \mathrm{~s}(1,7)=8.55$ and 18.63 , ps $<.025$ and .005 , respectively]; neither of the corresponding residual nonlinear components was significant, $[F \mathrm{~S}(4,28)=.74$ and 1.10, n.s. $]$. The effect of the factor target/no-target was marginally significant $[F(1,7)=$ $5.44, p=.05]$, with the no-target condition taking longer than the target condition. Although the interaction term, number of items $\times$ target/no-target, was not statistically significant $[F(5,35)=1.12, p=.37[$, the slope of the no-target function was $196\left(r^{2}=.88\right)$, while that of the target function was $122\left(r^{2}=.88\right)$.

The reaction times for the six different display conditions are all considerably higher than those for the corresponding orientation conditions of the disjunction task.

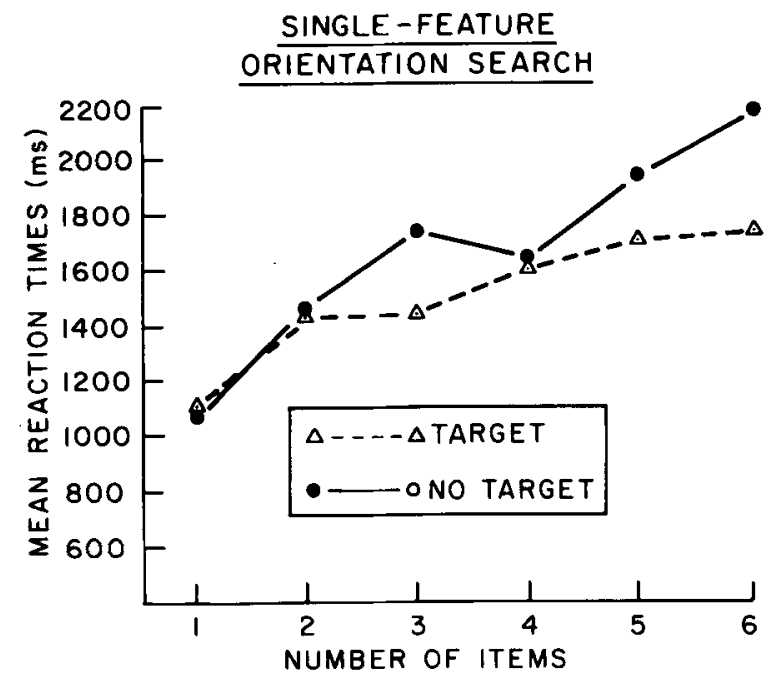

Figure 4. Experiment 3: Mean search times as a function of number of items in the stimulus display (single-feature orientation search task).
The associated standard deviations and percent errors (presented in Table 1), are also a great deal larger, providing additional evidence that the manipulation of task difficulty was successful. The nonsignificant number of items $\times$ target/no-target interaction term may simply reflect reduced power in this experiment, due to higher response times and associated higher variability. Given that the ratio of no-target to target slopes was 1.61 , almost identical to that obtained in Experiment 2 (which was not statistically different from 2.0 ), it does not seem plausible to accept the null hypothesis.

In generally satisfying the predictions above regardin an increase in reaction times and a slope ratio greater than $1: 1$, the data may be reasonably interpreted as providing a haptic counterpart of memory search with visual probe. (Sternberg, 1975): A difficult haptic search task causes the use of a serial self-terminating search process. However, the implications of these results for interpreting Experiment 1 are not entirely straightforward. One line of reasoning is to assume that the difference between Experiment 1 (easy disjunctive) and Experiment 3 (difficult single-feature) is like that observed with memory search: it represents a shift from serial exhaustive to selfterminating processing as search rate is slowed. However, self-termination in Experiment 3 does not definitively establish exhaustive scanning in Experiment 1. The two studies may still represent distinct processing mechanisms, namely parallel and serial.

In addition, these results differ from those of Experiment 2 (conjunctive). Experiments 1 and 2 showed approximately equal reaction times to single-item displays, as is expected for disjunctive and conjunctive tasks; in Experiment 3, performance was considerably slower. On this basis, the difficulty manipulation of Experiment 3 appears to involve single-item processing (i.e., a difficulty in discriminating between target and distractors, requiring serial fixation of each item with the appropriate finger-"haptic foveation"); in contrast, conjunctive search follows the pattern predicted when difficulty occurs because of the need to verify a common spatial locus for two discrete object dimensions (see Treisman \& Gelade, 1980, p. 113). The difference in what constitutes task difficulty again suggests caution in using Experiment 3 to infer the nature of disjunctive-task processing.

\section{GENERAL DISCUSSION}

The results of these experiments can be summarized as follows: In a disjunctive search task with texture or orientation targets, haptic observers produced reaction time functions that increased nonlinearly with items and had equal slopes for target and no-target conditions. In a conjunctive version of the task, the functions were linear (notarget) or nearly so (target), the slope ratio was $2: 1$, and both functions increased more rapidly than in the disjunction task. There was no difference in reaction times for the one-item searches in the two experiments. Finally, a difficult single-feature search for orientation also produced 
very steep linear functions with a slope ratio close to $2: 1$; overall, the reaction times, standard deviations, and errors were considerably greater than those obtained in either the disjunction or conjunction tasks.

In keeping with Treisman's feature integration theory, we suggested that disjunctive search for simple orientation and texture targets might use a parallel, capacityunlimited process, on which was superimposed an additional, at least partially serial, process of finger alignment. This would lead to the nonlinear, parallel increasing functions observed in that task, under the assumptions outlined above. A second alternative is that the parallel functions of the disjunction task represent the use of a serial exhaustive search process. This would seem particularly appropriate if the task could be more efficiently performed using exhaustive, as opposed to self-terminating, processing. Unfortunately, the current data do not permit us to decide conclusively in favor of either interpretation at this time. Nevertheless, these data show clear contrasts in the haptic processing of information under different search demands (disjunctive, conjunctive, and difficult singlefeature search).

The results of Experiments 2 and 3 further address these two interpretations. We have noted that the reaction time slope ratios obtained in Experiments 2 and 3 were both close to $2: 1$. It appears, then, that haptics used a serial self-terminating attentive process in both the conjunction and single-feature search tasks. Clearly, task difficulty is an important factor in eliciting haptic, as well as visual, serial self-terminating attentive processing (at least when processing orientation; recall that texture was not tested). However, we have noted that in Experiment 3, the reaction times were generally slow. And more specifically, single-item response times were larger than those of Experiment 1, whereas in Experiment 2, the single-item response times matched those of Experiment 1. This suggests that Experiment 3 was difficult because of low interitem discriminability, whereas Experiment 2 follows the pattern predicted when difficulty arises because of the need to focus attention on spatially distributed information. Furthermore, the disjunctive search in Experiment 1 might have elicited parallel processing, as Treisman has demonstrated in corresponding visual tasks.

We view the present results as a first step in determining the nature of early haptic processing. At this point, it is not known which haptic primitives, will prove most valuable in the development of models of human and machine haptic object processing. We use the term "primitives" in the computational modeling sense (e.g., Marr, 1982), to refer to the simplest components in an object representation. Doubtless, there will be different primitives at different levels, as is common in computational vision systems (e.g., Marr, 1982). There are a number of criteria that could be used to judge if a given dimension should be included as a haptic primitive. Unfortunately, none is universally accepted. In the cur- rent paradigm, Treisman's "pop-out" phenomenon (i.e., detection time invariant over display size), would arguably be the strongest evidence, in that it suggests that dimensional information is detected automatically. If the present disjunctive search pattern is taken as evidence of underlying parallel processing, both line orientation and surface roughness would be candidates for primitives. However, the equivocal nature of the results precludes such a conclusion.

There are several reasons why texture, more than orientation, would seem to be a reasonable haptic primitive candidate, in the sense that it is a dimension that could be processed preattentively. At the time the research was initiated, there was very little in the literature to guide the choice of potential haptic primitives. In keeping with much of the earlier tactile work, our selection of orientation was dictated primarily by the fact that it was commonly used in computational, neurophysiological, and behavioral models of visual pattern perception. There was a stronger basis for the selection of texture, given not only the computational and behavioral models that existed for this dimension, but also psychophysical evidence that texture can be processed relatively quickly and accurately. Subsequent work suggests, however, that information about texture is readily available haptically, whereas information about orientation is not.

One relevant body of work is that of Loomis (1981), who has modeled cutaneous extraction of shape information as bandwidth(resolution)-limited vision. The resolving power of the fingertip for orientation is sufficiently limited to cast doubt on a preattentive mechanism that could make even modestly fine orientation discriminations. A second set of findings comes from research of Lederman and Klatzky (1987; Klatzky et al., 1987), who have found that extraction of precise contour beyond the scale of the fingertip requires a temporally extended period of contour exploration that is relatively error prone. Klatzky et al. further found that, relative to vision and haptics used together, haptic exploration alone led to low salience for shape properties of objects and high salience for the substance properties of texture and hardness. Haptic explorers without vision tended to eschew the extensive contour examination needed to determine shape precisely. The necessity for such exploration may motivate serial attentive search, as was evidenced in our Experiment 3. Finally, a third source of converging results that contrast the processing of texture and orientation derives from pilot work for the current study. In single-feature and disjunctive search tasks with simple discriminations, the texture results consistently showed low slopes, whereas the results for orientation were considerably more labile and the slopes tended to have higher values.

Clearly, in developing a haptic analogue to Marr's (1982) work on computer vision, further research is necessary to determine the units that are combined at various levels of haptic processing. By establishing qualitative 
differences in haptic disjunctive and conjunctive search, the present results represent an initial first step toward this goal.

\section{REFERENCES}

Craig, J. (1985). Attending to two fingers: Two hands are better than one. Perception \& Psychophysics, 38, 496-511.

GiBson, J. J. (1966). The senses considered as perceptual systems. Boston: Houghton-Mifflin.

HARMON, L. D. (1982). Automated tactile sensing. Intemational Journal of Robotics Research, 1, 3-32.

Klatzky, R. L., Lederman, S. J., Metzger, V. (1985). Identifying objects by touch: An "expert system." Perception \& Psychophysics, 37, 299-302.

KLAtZKY, R. L., Lederman, S. J., REED, C. (1987). There's more to touch than meets the eye: The relative salience of object dimen. sions for haptics with and without vision. Joumal of Experimental Psychology: General, 116, 356-369.

KRUEGER, L. (1984). Self-termination in same-different judgments: Multiletter comparison with simultaneous and sequential presentation. Journal of Experimental Psychology: Leaming, Memory, \& Cognition, 10, 271-284

Krueger, L., \& Shapiro, R. (1980). Why search for target absence is so slow (and careful!): The more targets there are, the more likely you are to miss one. Journal of Experimental Psychology: Human Perception \& Performance, 6, 662-685.

LAMB, G. (1983). Tactile discrimination of textured surfaces: Psychophysical performance measurements in humans. Joumal of Physiology, 338, 551-565.

LAPPIN, J., \& FoulKe, E. (1973). Expanding the tactual field of view, Perception \& Psychophysics, 14, 237-241.

Lederman, S. J. (1982). The perception of texture by touch. In W. Schiff \& E. Foulke (Eds.), Tactual perception: A sourcebook (pp. 130-167). New York: Cambridge University Press.

Lederman, S. J., ABbott, S. G. (1981). Texture perception: Studies of intersensory organization using a discrepancy paradigm and visual vs tactual psychophysics. Journal of Experimental Psychology: Human Perception \& Pefformance, 7, 902-915.

Lederman, S. J., KLATZKy, R. L. (1987). Hand movements: A window into haptic object recognition. Cognitive Psychology, 19, 342-368.

Loomis, J. (1981). On the tangibility of letters and braille. Perception \& Psychophysics, 29, 37-46.

LoOmis, J. M., \& Lederman, S. J. (1986). Tactual perception. In K. Boff, L. Kaufman, \& J. Thomas (Eds.), Handbook of perception and human performance (Vol. 2, chap. 31, pp. 1-41). New York: Wiley.

MARR, D. (1982). Vision: A computational investigation into the human representation and processing of visual information. San Francisco: Freeman.
Morely, J., Goodwin, A., Darian-Smith, I. (1983). Tactile discrimination of gratings. Experimental Brain Research, 49, 291-299.

Nickerson, R. S. (1966). Response times with a memory-dependent decision task. Joumal of Experimental Psychology, 72, 761-769.

Sherrick, C., Cholewiak, R. (1986). Cutaneous sensitivity. In K. Boff, L. Kaufman, \& J. Thomas (Eds.), Handbook of perception and human performance Vol. I, 12(1-58). New York: Wiley.

Shiffrin, R., Craig, J., Cohen, E. (1973). On the degree of attention and capacity limitation in tactile processing. Perception \& Psychophysics, 13, 328-336.

Sterngerg, S. (1975). Memory scanning: New findings and current controversies. Quarterly Journal of Experimental Psychology, 27. 1-32.

TownSEnd, J. T. (1974). Issues and models concerning the processing of a finite number of inputs. In B. Kantowitz (Ed.), Human information processing: Tutorials in performance and cognition (pp. 133-185). Hillsdale, NJ: Erlbaum.

Townsend, J., Ashby, G. (1983). Stochastic modeling of elementary psychological processes. New York: Cambridge University Press.

Treisman, A. (1986). Properties, parts, and objects. In K. Boff, L. Kaufman, \& J. Thomas (Eds.), Handbook of perception and human performance Vol. II, 35(1-70). New York: Wiley.

Treisman, A., Gelade, G. (1980). A feature integration theory of attention. Cognitive Psychology, 12, 97-136.

Treisman, A., \& Paterson, R. (1984). Emergent features, attention and object perception. Joumal of Experimental Psychology: Human Perception \& Performance, 10, 12-31.

TREISMAN, A., SCHMidT, H. (1982). Hlusory conjunctions in the perception of objects. Cognitive Psychology, 14, 107-114.

Treisman, A., Sykes, M., Gelade, G. (1977). Selective attention and stimulus integration. In S. Dornik (Ed.), Attention and performance, VI (pp. 333-361). Hillsdale, N.J.: Erlbaum.

\section{NOTE}

1. Similar nonlinearities might or might not be expected to appear in the conjunction data. A linear function could be interpreted as indicating that subjects chose to align each finger and process each item in a strictly serial fashion, knowing the nature of the conjunction task ahead of time. Nonlinearities in the function could indicate that subjects prealigned fingers (as was proposed for the disjunctive task), then performed a serial comparison process. The data suggest, in fact, that both approaches may have been taken: There were some, but only marginally significant, nonlinearities in the conjunction target function, and none in the no-target function.

(Manuscript received June 1, 1987; revision accepted for publication January $29,1988$. ) 